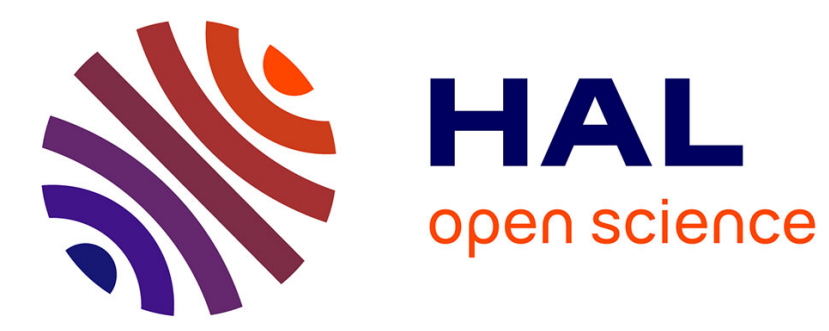

\title{
Precision Prediction Using Interval Exponential Mapping of a Parallel Kinematic Smart Composite Microstructure.
}

Sergio Andree Lescano Alvarado, Micky Rakotondrabe, Nicolas Andreff

\section{- To cite this version:}

Sergio Andree Lescano Alvarado, Micky Rakotondrabe, Nicolas Andreff. Precision Prediction Using Interval Exponential Mapping of a Parallel Kinematic Smart Composite Microstructure.. RSJ International Conference on Intelligent Robots and Systems, Sep 2015, Hamburg, Germany. hal-02868200

\section{HAL Id: hal-02868200 https://hal.science/hal-02868200}

Submitted on 15 Jun 2020

HAL is a multi-disciplinary open access archive for the deposit and dissemination of scientific research documents, whether they are published or not. The documents may come from teaching and research institutions in France or abroad, or from public or private research centers.
L'archive ouverte pluridisciplinaire HAL, est destinée au dépôt et à la diffusion de documents scientifiques de niveau recherche, publiés ou non, émanant des établissements d'enseignement et de recherche français ou étrangers, des laboratoires publics ou privés. 


\title{
Precision Prediction Using Interval Exponential Mapping of a Parallel Kinematic Smart Composite Microstructure
}

\author{
Sergio Lescano, Micky Rakotondrabe, member, IEEE, and Nicolas ANDREFF, member, IEEE
}

\begin{abstract}
In this paper, a method to predict the precision of parallel microstructures made of carbon fibers, polyimide layers and piezoelectric materials is proposed. To this aim, we propose to combine the exponential representation of transformation matrix with intervals to bound the uncertainties in each flexure joint of the microstructures. Based on interval techniques and algorithms, the derived model permits to guarantee a bound on the precision value of the end effector. The method has been demonstrated with a numerical example of parallel microrobot devoted to orient the laser spot towards vocal fold during phonomicrosurgery. This example considers the uncertainties that occur in the compliant joints caused by the fabrication technique.
\end{abstract}

\section{INTRODUCTION}

Phonomicrosurgery ${ }^{1}$ with laser was introduced by Jako [1] in the 1970's. Even though there exist a variety of laser technologies, the prevalent traditional methodology for remote control of the laser is a mechanical manipulator. However, many of the difficulties associated in using this classical mechanical manipulator are rooted in the ergonomics of the device, thereby decreasing the quality of the intervention.

Within the European $\mu$ RALP project [2], a fiber guides the laser from its external (proximal) source to the distal tip of the laryngoscope, where a robotic micromanipulator is placed to precisely orient the laser beams (Fig. 1a). The laryngoscope diameter is $20 \mathrm{~mm}$, and the space assigned for the microrobot is $10 \times 10 \times 10 \mathrm{~mm}^{3}$. The distal tip of the laryngoscope is introduced into the larynx, at a distance up to $20 \mathrm{~mm}$ from the vocal fold as depicted in Fig. 1b. The microrobot, which is proposed in this paper, is a parallel kinematics manipulator (PKM) with two rotational degreesof-freedom (dof). A micromirror is placed on its mobile platform in order to reflect the laser beam. The PKM microrobot is specified to have a minimal bandwidth of $200 \mathrm{~Hz}$ and a scanning resolution better than $100 \mu \mathrm{m}$ in order to prevent overheating and damaging of the healthy tissue. Piezoelectric materials with cantilever structures $\left(10 \times 2 \mathrm{~mm}^{2}\right)$ are used to actuate the microrobot because of the very high resolution

This work has been supported by the European Union Seventh Framework Programme. Project "Micro-Technologies and Systems for Robot assisted Laser Phonomicrosurgery" called $\mu$ RALP - www . microralp.eu - (FP7ICT-2011-7), and by the French ANR Labex no. ANR-11-LABX-01-01 (www.labex-action.fr)

The authors are with FEMTO-ST Institute, CNRS UMR 6174 UFC / ENSMM / UTBM, Automatic Control and Micro-Mechatronic Systems Department, 25000 Besançon, France \{sergio.lescano, mrakoton, nicolas.andreff\}efemto-st.fr

${ }^{1}$ Phonomicrosurgery: Set of medical procedures used to treat abnormalities on the vibratory elements of the vocal fold in order to restore or improve voice function.

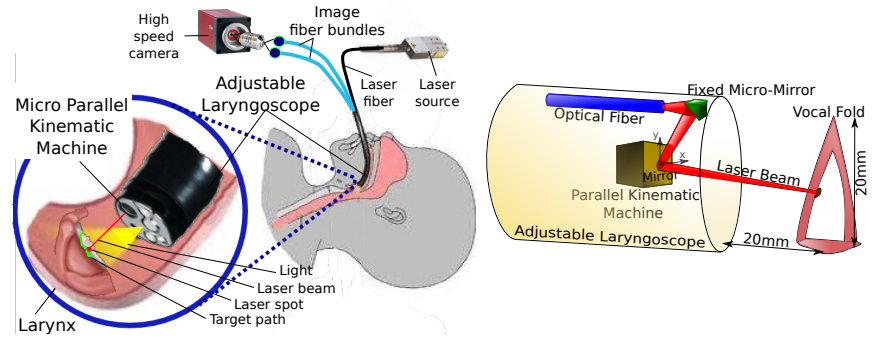

(a)

(b)

Fig. 1: (a) Endoscopic laser phonomicrosurgery ( $\mu$ RALP project [2]). (b) Principle of the micro-PKM placed at distal tip of the laryngoscope and steering the laser beam onto the vocal fold [4].

(nanometric), the large bandwidth (up to several $\mathrm{KHz}$ ) and the high force density they can offer [3].

To create the complex three-dimensional (3D) structure at small scale, we turned ourselves to the recent origamiinspired techniques [5], where flexible and rigid materials are combined in one monolithic flat structure which is then folded in order to obtain a complex and stiff mechanical structure. A specific origami-inspired technique called Smart Composite Microstructure (SCM) permits to combine piezoelectric materials, carbon fibers and polymer (polyimide) materials at small sizes [6], [7]. The major advantages of SCM microfabrication technology are its simplicity for making small 3D mechanisms and its ability to create flexure joints with larger range of motion than those made in silicon. Moreover, SCM technique yields remarkable improvements without causing either fracture or rapid fatigue on the operating structure. Several structures have been created using this technique [8], [9], [10] including structures that can spontaneously reach 3D shapes, called self-folding machines [11].

Due to the SCM fabrication process and by the nature of the materials used, uncertainties in angles, axis directions and point positions are observed in the flexure joints. These uncertainties greatly influence the precision of the endeffector. In fact, a small uncertainty in one joint is amplified and transmitted by the rest of the structure till the endeffector (uncertainties propagation). One advantage to use a parallel structure is that uncertainties generated by each individual leg can be filtered out by the kinematic constraints imposed by the other legs. Unfortunately, not all uncertainties can be cancelled and a method for estimating the residual end-effector uncertainty is needed. 


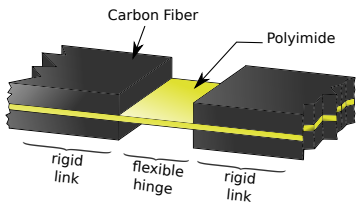

(a)

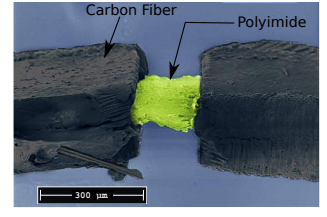

(b)
Fig. 2: (a) CAD model of a hinge using SCM fabrication technique. (b) Pseudo-colored SEM image of a fabricated hinge (by SCM) depicting the carbon fiber along with the flexible polyimide $($ scale $=300 \mu \mathrm{m})$.

To do so, we chose to use interval analysis because it is pessimistic in nature and does not require any statistical model of the uncertainties. Interval analysis was applied to parallel robots (e.g. in [12]) to solve the forward kinematic problem and to ensure the reliability of the robot, among others. Also, in [13][14] a methodology is explained to ensure the precision of serial structures via interval analysis using the exponential mapping for rigid motion.

The contributions of this paper are as follows. First, the uncertainties caused by the SCM fabrication technique on flexure joints are presented (Section II). Then, a methodology to calculate the uncertainties at the end-effector is described (Section III). Its novelty lies in the application to PKM of the interval exponential mapping used in [13][14] for serial structures. Finally, a novel microrobot is introduced and its precision analysed to exemplify the methodology (Section IV).

\section{UNCERTAINTIES DUE TO THE SCM FABRICATION TECHNIQUE}

A simple flexure joint made with the SCM process is shown in Fig. 2, where three parts are distinguished: rigid flexible - rigid, as depicted in Fig. 2a. Basically the flexible part is used to fold the structure and the rigid part serves as a link. A Scanning Electron Microscope image of a fabricated sample at our facility is shown in Fig. 2b. It is based on one polyimide layer that serves as a flexible element and two carbon fiber layers to stiffen the structure. The thickness of each carbon fiber layer and polyimide layer is $180 \mu \mathrm{m}$ and $10 \mu \mathrm{m}$, respectively.

A bent joint of the fabricated SCM microstructure is shown in Fig. 3a. As shown in Fig. 3b, the joint itself generates undesired strains. Thus, in each joint, SCM microfabrication results in an uncertain Euclidean transformation built upon three components:

- uncertainties on the joint angle,

- uncertainties on the joint axis direction,

- uncertainties on the position of the joint axis.

that can be quantified from the dimensions of the flexible hinge: the rigid-to-rigid distance for the position or the cone defined by the hinge (Fig. 3b) for the direction.

Thanks to interval analysis, we do not need to push further the modeling of the associated physics and can rely on (interval) kinematic representations of the latter components.

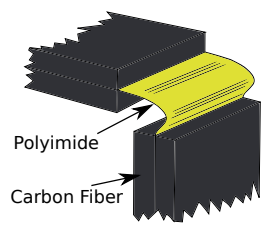

(a)

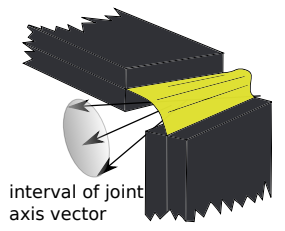

(b)
Fig. 3: (a) A hinge based on the flexibility of a polyimide material. (b) Sprained kink dislocating the rotation axis in position and direction.

\section{UNCERTAIN KINEMATIC ANALYSIS OF SCM USING INTERVAL ANALYSIS}

\section{A. Spatial rigid motion tools}

In this section, we propose to combine the exponential mapping and interval tools to highlight the uncertain motions that cannot be directly handled by other representations. In fact, exponential representation allows a global description and a geometric handling of spatial rigid body motions and is widely described in classically sized robotic systems [15]. Thus, an exponential mapping representation for a rigid motion in $S E(3)$ (special Euclidean group) is used in order to account for the uncertain motions present in the joints.

Remind that the motion generated by a rotational joint is described by the homogeneous transformation $T_{\text {joint }} \in$ $S E(3)$ obtained by the following matrix exponential:

$$
T_{\text {joint }}=\mathrm{e}^{\hat{\boldsymbol{\xi}} q}
$$

where $q$ is the joint variable and $\hat{\boldsymbol{\xi}}$ belongs to $s e(3)$, the Lie algebra associated to $S E(3)$. The latter is formed as

$$
\hat{\boldsymbol{\xi}}=\left[\begin{array}{cc}
\hat{\boldsymbol{\omega}} & -\boldsymbol{\omega} \times \boldsymbol{p}_{\text {joint }} \\
0 & 0
\end{array}\right]
$$

where $\boldsymbol{p}_{\text {joint }}$ is the joint position and

$$
\hat{\boldsymbol{\omega}}=\left[\begin{array}{ccc}
0 & -\omega_{3} & \omega_{2} \\
\omega_{3} & 0 & -\omega_{1} \\
-\omega_{2} & \omega_{1} & 0
\end{array}\right]
$$

where $\hat{\boldsymbol{\omega}} \in \operatorname{so}(3)$ is built upon $\boldsymbol{\omega}=\left[\begin{array}{lll}\omega_{1} & \omega_{2} & \omega_{3}\end{array}\right]^{T}$ the unitary vector representing the direction of the joint axis.

\section{B. Intervals as representation of the uncertainties}

Let us express the joint variables, axis directions and position coordinates by intervals as follows:

- a (scalar) joint variable $q$ becomes $Q=\left[q-\delta_{q}, q+\delta_{q}\right]$;

- a joint axis direction $\boldsymbol{\omega}=\left[\begin{array}{lll}\omega_{1} & \omega_{2} & \omega_{3}\end{array}\right]^{T}$ becomes

$$
\boldsymbol{\Omega}=\left[\begin{array}{l}
\boldsymbol{\omega}_{1}-\delta_{\omega_{1}}, \boldsymbol{\omega}_{1}+\delta_{\omega_{1}} \\
\boldsymbol{\omega}_{2}-\delta_{\omega_{2}}, \boldsymbol{\omega}_{2}+\delta_{\omega_{2}} \\
\boldsymbol{\omega}_{3}-\delta_{\omega_{3}}, \boldsymbol{\omega}_{3}+\delta_{\omega_{3}}
\end{array}\right]
$$

- a joint position $\boldsymbol{p}=\left[p_{x}, p_{y}, p_{z}\right]^{T}$ becomes

$$
\boldsymbol{P}=\left[\begin{array}{l}
\boldsymbol{p}_{x}-\delta_{p_{x}}, \boldsymbol{p}_{x}+\delta_{p_{x}} \\
\boldsymbol{p}_{y}-\delta_{p_{y}}, \boldsymbol{p}_{y}+\delta_{p_{y}} \\
\boldsymbol{p}_{z}-\delta_{p_{z}}, \boldsymbol{p}_{z}+\delta_{p_{z}}
\end{array}\right]
$$




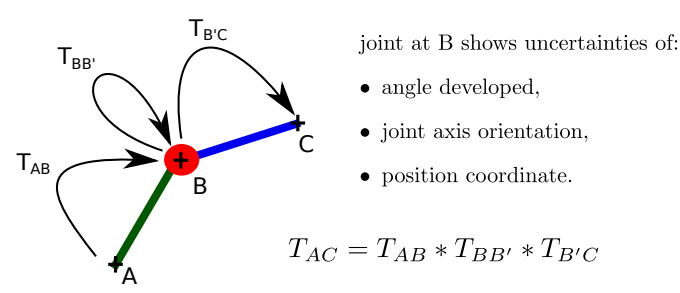

Fig. 4: Two links and a flexible hinge at $B$ with uncertainties due to the SCM fabrication technique.

where $\delta_{i}$, with $i \in\left\{q, \omega_{1}, \omega_{2}, \omega_{3}, p_{x}, p_{y}, p_{z}\right\}$, correspond to the radius of uncertainties.

\section{Transfering the uncertainties}

Similar to Fig. 3b, Fig. 4 shows two rigid links attached by the joint in B. Uncertainties due to the SCM fabrication technique are exhibited at the joint B (uncertainties of angle, axis direction and position).

Thus, the homogeneous transformation between the reference frame in $\mathrm{A}$ and the one in $\mathrm{C}$ is denoted by: $T_{A C}=$ $T_{A B} * T_{B B^{\prime}} * T_{B^{\prime} C}$, where the homogeneous transformation $T_{B B^{\prime}}$ takes into account the joint uncertainties in B. It is explicitly denoted as:

$$
T_{B B^{\prime}}=\mathrm{e}^{\hat{\Xi}_{B} Q_{B}}
$$

where $\hat{\boldsymbol{\Xi}}_{B}$ is an interval matrix that is compatible with the structure of $\operatorname{se}(3)$ :

$$
\hat{\boldsymbol{\Xi}}_{B}=\left[\begin{array}{cc}
\hat{\boldsymbol{\Omega}}_{B} & -\boldsymbol{\Omega}_{B} \times \boldsymbol{P}_{B} \\
0 & 0
\end{array}\right]
$$

$Q_{B}$ is the interval representing the uncertain angle developed at B. $\Omega_{B}$ is the vector interval representing the uncertain axis direction of the joint at B. $\boldsymbol{P}_{B}$ is the vector interval representing the uncertain position of the point $\mathrm{B}$.

\section{Methodology to evaluate uncertainties in parallel robots}

Our methodology to find the uncertainties in the platform follows the following steps.

- The analysis starts by solving the inverse kinematics of the parallel robot, in a given configuration using conventional arithmetics.

- Then, the uncertainties corresponding to each joint $(\boldsymbol{q} \rightarrow \boldsymbol{Q}$ and $\boldsymbol{\xi} \rightarrow \boldsymbol{\Xi})$ are introduced, using the interval exponential matrices.

- Finally, the implicit kinematic constraint is solved for the end-effector pose $\boldsymbol{X}$, given the calculated uncertainties.

a) Inverse Kinematics: It deals with computing the joint variables $q$ with respect to the operational angles $x$. In [16], a methodology to calculate the inverse kinematics of a parallel robot using the implicit equations of the structure geometry was described. Initially, the coordinates of one specific point on the robot are expressed as a function $H_{1}(x)$ of the end-effector variables. Then, the same coordinates are expressed as a function $\mathrm{H}_{2}(q)$ of the joint variables. Thereby, equating $H_{1}(x)$ and $H_{2}(q)$ yields the implicit kinematic constraint: $H_{1}(x)=H_{2}(q)$. Finally, joint variables are expressed in function of the end-effector variables $(q=f(x))$.

b) Exponential Interval Matrices: After taking into account uncertainties of the joint variables (position, direction and angle), the evaluation of the overall precision is based on exponential interval matrices.

Nevertheless, the counterpart of the easy representation of uncertainties with intervals parameters is the numerical calculation of the exponential of interval matrix. Goldsztejn [17] proposed a computational method to calculate the exponential of an interval matrix, based on the truncated Taylor or Padé expansion (Horner-Taylor series) computed in double precision. Among the three methods to calculate such an exponential, this method minimizes the overestimation in the results [17]. This method is therefore employed here as it is well adapted to precision evaluation.

c) Solving the uncertain implicit kinematic constraint: The problem which consists in finding the set $X$ of variable $x$ such that $f(x) \in Y$, where $Y$ is a given set, is a set inversion problem. That is, find $X$ such that $X=$ $\left\{x \in \Re^{n} \mid f(x) \in Y\right\}=f^{-1}(Y)$. Such a problem can be solved by a set inversion algorithm, such as SIVIA (set inversion via interval analysis)[18]. This algorithm is based on interval techniques and operations and is summarized in Table-1, where $w([\mathbf{x}])$ is the width of the interval argument $[\mathbf{x}]$ and $\epsilon$ is the accuracy of the computation. $\mathbb{S}$ and $\overline{\mathbb{S}}$ correspond to the upper and lower bounds of the set solution $\mathbb{S}: \underline{\mathbb{S}} \subseteq \mathbb{S} \subseteq \overline{\mathbb{S}}$.

TABLE I: The SIVIA algorithm [18].

\begin{tabular}{|l|l|}
\hline & SIVIA(inputs: $[\mathbf{x}], \epsilon ;$ outputs: $\underline{\mathbb{S}}, \overline{\mathbb{S}})$ \\
\hline 1 & if $[\mathbf{f}]([\mathbf{x}]) \cap[\mathbf{Y}]=\emptyset,[\mathbf{x}]$ is non-solution; \\
2 & if $[\mathbf{f}]([\mathbf{x}]) \subseteq[\mathbf{Y}]$, thus $\underline{\mathbb{S}}:=\underline{\mathbb{S}} \cup[\mathbf{x}] ; \overline{\mathbb{S}}:=\overline{\mathbb{S}} \cup[\mathbf{x}] ;$ \\
3 & if $w([\mathbf{x}])<\epsilon$ thus $\overline{\mathbb{S}}:=\overline{\mathbb{S}} \cup[\mathbf{x}] ;$ \\
4 & otherwise bisect $[\mathbf{x}]$ into $\left[\mathbf{x}_{1}\right]$ and $\left[\mathbf{x}_{2}\right] ;$ \\
& SIVIA(inputs: $\left[\mathbf{x}_{1}\right], \epsilon ;$ outputs: $\left.\underline{\mathbb{S}}, \overline{\mathbb{S}}\right) ;$ \\
& SIVIA(inputs: $\left[\mathbf{x}_{2}\right], \epsilon ;$ outputs: $\left.\underline{\mathbb{S}}, \overline{\mathbb{S}}\right)$. \\
\hline
\end{tabular}

In practice, SIVIA is used here together with the implicit kinematic constraints, that is a vector equation. Its interval solution is therefore the intersection of the sets obtained by solving each coordinate independently.

\section{IMPLEMENTATION OF AN EXAMPLE}

In this section, we introduce a novel parallel kinematics microrobot, fabricated by the SCM technique, and we perform its precision analysis.

\section{A. Kinematic description}

In a previous work [4], we performed the complete kinematic analysis of the proposed kinematic structure (Fig. 5) using screw theory and considering perfect joints and undeformable links of the microrobot. The mobile platform of the latter holds a micromirror used to reflect the laser 


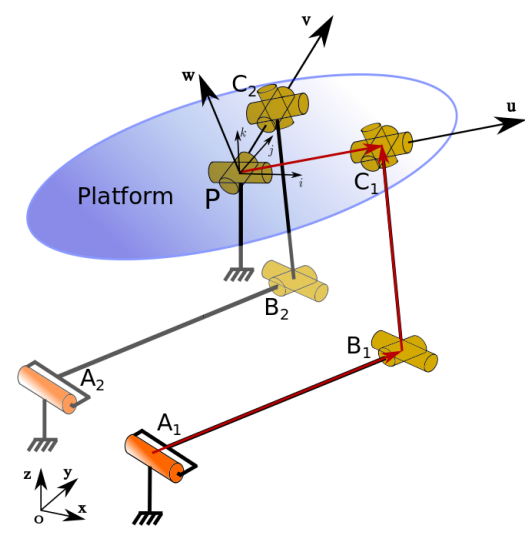

Fig. 5: Platform operated in parallel by two active legs and constrained by an passive U-joint leg [4].

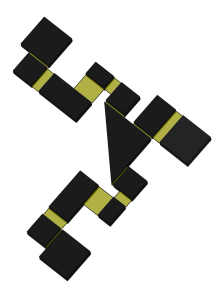

(a) CAD design.

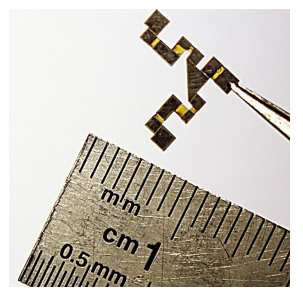

(b) Realization.
Fig. 6: Planar structure.

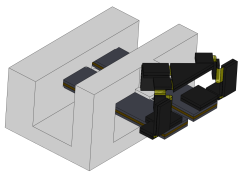

(c) CAD design.

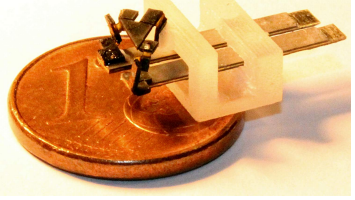

(d) Realization.
Fig. 7: Folded 3D mechanism

beam, which can rotate about the $u$-axis and the $v$-axis. The end-effector is connected to the base by a passive U-joint at point $P$ (Fig. 5) allowing only 2 rotations. The end-effector is actuated with two identical 6-dof RUS legs.

In the reference configuration, the angles of the mobile platform are $\theta_{1}=\theta_{2}=0$. Consequently, $\mathbf{c}_{1}=\overrightarrow{P C_{1}}$ is parallel to $\mathbf{u}=\mathbf{i}$ and $\mathbf{c}_{2}=\overrightarrow{P C_{2}}$ is parallel to $\mathbf{v}=\mathbf{j}$.

\section{B. Fabrication}

The SCM technique was used to make the parallel kinematic microrobot. A planar structure, as pictured in Fig. 6 (CAD model and photo of the fabrication), was first fabricated. It contains the passive U-joints (points $P$ and $B_{j}$ ) and S-joints (points $C_{j}$ ). Then it is folded to obtain a 3D structure. After folding, two piezoelectric cantilever actuators are glued to the two extremities of the folded structure. The two actuators correspond to the rods $A_{1} B_{1}$ and $A_{2} B_{2}$ of the Fig. 5 whilst the folded structure corresponds to the platform and the vertical legs. Fig. 7 depicts the CAD design and the assembled and fabricated microrobot respectively. The base and support were 3D printed.

\section{Inverse kinematics}

By observing Fig. 5 and given a feasible platform orientation, defined by the values of the passive-leg U-joint angles $\left(\theta_{1}, \theta_{2}\right)$, the mechanism configuration can be calculated by finding the intersection of a sphere centered at the point $C_{i}$, $i=1,2$, with radius $\left|B_{i} C_{i}\right|$ and the circle with center $A_{i}$, with radius $\left|A_{i} B_{i}\right|$, in a plane normal to the line $A_{1} A_{2}$. Thus, to find the implicit equation of the PKM shown in Fig. 5 we proceed as follows. On the one hand,

$$
\overrightarrow{P C_{i}}=\left.\left[\begin{array}{cc}
R\left(\theta_{1}, \theta_{2}\right) & 0_{3 \times 1} \\
0_{1 \times 3} & 1
\end{array}\right] \overrightarrow{P C_{i}}\right|_{u v w}=H_{1}\left(\theta_{1}, \theta_{2}\right)
$$

where $\overrightarrow{P C_{i}}$ and $\left.\overrightarrow{P C_{i}}\right|_{\text {uvw }}$ are the homogeneous coordinates representing the vectors linking $P$ and $C_{i}$ expressed in the reference system fixed at the point $P$ parallel to $x, y, z$ and $u, v, w$ respectively, and $R$ is the rotation matrix given by:

$$
R\left(\theta_{1}, \theta_{2}\right)=R_{x}\left(\theta_{1}\right) R_{y}\left(\theta_{2}\right)=\left[\begin{array}{ccc}
c_{2} & 0 & s_{2} \\
s_{1} s_{2} & c_{1} & -s_{1} s_{2} \\
-c_{1} s_{2} & s_{1} & c_{1} c_{2}
\end{array}\right]
$$

where $c_{1}=\cos \theta_{1}, s_{1}=\sin \theta_{1}, c_{2}=\cos \theta_{2}, s_{2}=\sin \theta_{2}$. On the other hand,

$$
\overrightarrow{P C_{i}}=\overrightarrow{P A_{i}}+\overrightarrow{A_{i} B_{i}}+\overrightarrow{B_{i} C_{i}}=H_{2}\left(q_{i}\right)
$$

where $\overrightarrow{P A_{i}}, \overrightarrow{A_{i} B_{i}}$ and $\overrightarrow{B_{i} C_{i}}$ are the homogeneous coordinates representing the vectors linking the points $P$ and $A_{i}$, $A_{i}$ and $B_{i}$, and $B_{i}$ and $C_{i}$ respectively, expressed in the reference system fixed at the point $P$ and parallel to $x, y, z$. The vector $q_{i}$ contains the joint variables (passive and active) of each leg $i$.

Combining (6) with (8) yields the implicit kinematic constraint that links the joint angles $q_{i}$ versus to the operational angles $\theta_{1}$ and $\theta_{2}$ :

$$
H_{1}\left(\theta_{1}, \theta_{2}\right)=H_{2}\left(q_{i}\right)
$$

from which the inverse kinematics can be extracted:

$$
q_{i}=F_{i}\left(\theta_{1}, \theta_{2}\right)
$$

\section{Applying Uncertainties as Intervals}

Evaluating (10) in a given end-effector configuration and then imposing uncertainty intervals, our methodology yields an interval formulation of (8):

$$
\left[\overrightarrow{P C_{i}}\right]=\left[\overrightarrow{P A_{i}}\right]+\left[\overrightarrow{A_{i} B_{i}}\right]+\left[\overrightarrow{B_{i} C_{i}}\right]
$$

where square brackets denote interval vectors. Since the uncertainties of the fabrication of the base are constant,

$$
\left[\overrightarrow{P C_{i}}\right]=\left.T\left(\Theta_{1}, \Theta_{2}\right) \overrightarrow{P C_{i}}\right|_{u v w}
$$

where $\Theta_{1}$ and $\Theta_{2}$ are intervals denoting the uncertainties of the mobile platform orientation and

$$
T\left(\Theta_{1}, \Theta_{2}\right)=\left[\begin{array}{cc}
R\left(\Theta_{1}, \Theta_{2}\right) & 0_{3 \times 1} \\
0_{1 \times 3} & 1
\end{array}\right]
$$

is the interval matrix associated to (7). 


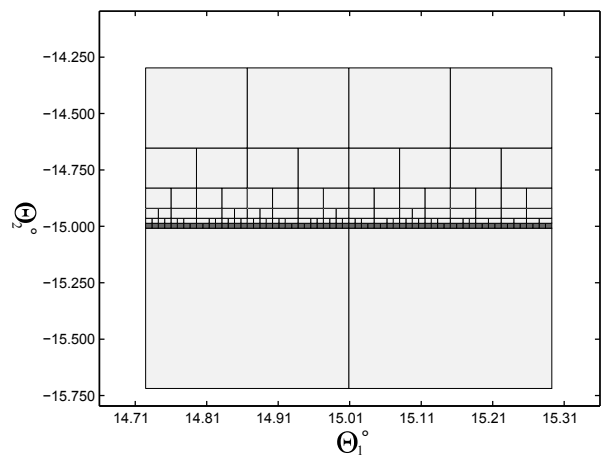

(a)

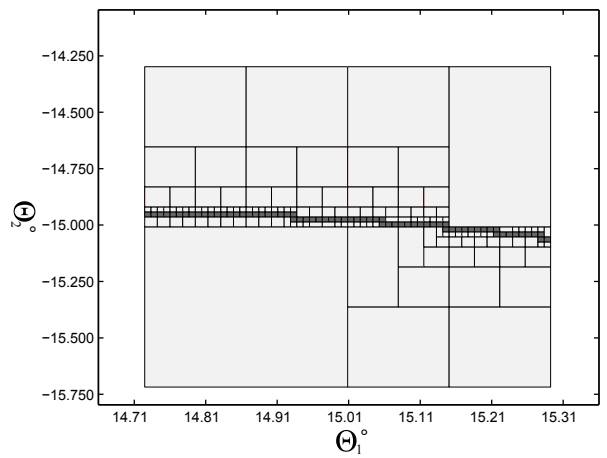

(c)

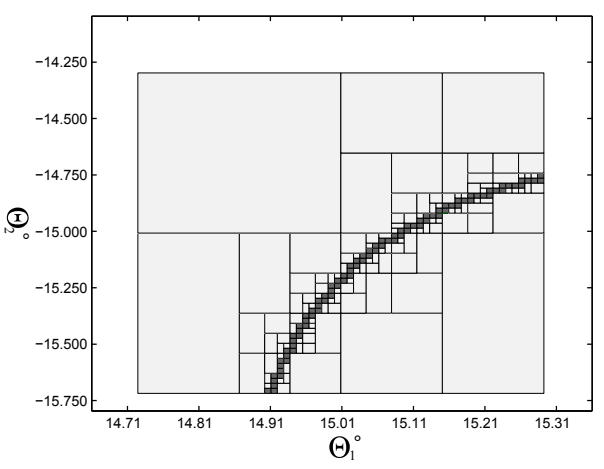

(b)

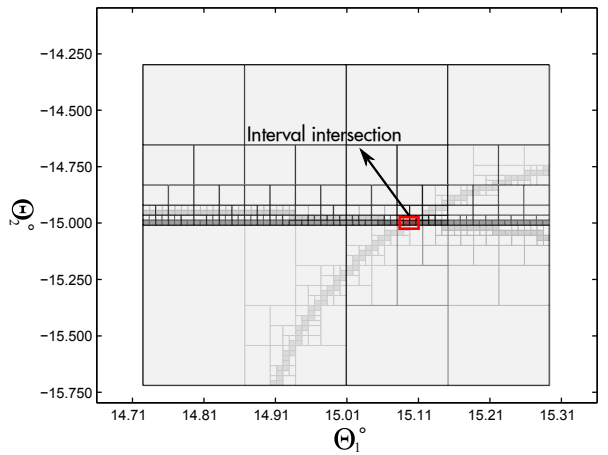

(d)

Fig. 8: Result of the components of (11) by SIVIA algorithm (a) of the first component. (b) of the second component. (c) of the third component. (d) Outcome of the intersection of all the three solutions shown above.

Furthermore, as $\overrightarrow{P A_{i}}$ are fixed points on the support of the robot and are not prone to uncertainties, we trivially have:

$$
\left[\overrightarrow{P A_{i}}\right]=\overrightarrow{P A_{i}}
$$

The remaining terms in (11) are expressed using the serial kinematic chain from the base to the S-joint on the platform:

$$
\begin{aligned}
{\left[\overrightarrow{A_{i} B_{i}}\right]=\left[B_{i}\right]-\left[A_{i}\right]=} & \left.T_{1}^{i} \mathrm{e}^{\hat{\Xi}_{1} Q_{1}^{i}} T_{2}^{i} \mathrm{e}^{\hat{\Xi}_{2} Q_{2}^{i}} \mathrm{e}^{\hat{\Xi}_{3} Q_{3}^{i}} B_{i}\right|_{B} \\
& -\left.T_{1} A_{i}\right|_{A} \\
{\left[\overrightarrow{B_{i} C_{i}}\right]=\left[C_{i}\right]-\left[B_{i}\right]=} & \left.T_{1}^{i} \mathrm{e}^{\hat{\Xi}_{1} Q_{1}^{i}} T_{2}^{i} \mathrm{e}^{\hat{\Xi}_{2} Q_{2}^{i}} \mathrm{e}_{3} Q_{3}^{i} C_{i}\right|_{B} \\
& -\left.T_{1}^{i} \mathrm{e}^{\hat{\Xi}_{1} Q_{1}^{i}} T_{2}^{i} \mathrm{e}^{\hat{\Xi}_{2} Q_{2}^{i}} \mathrm{e}^{\hat{\Xi}_{3} Q_{3}^{i}} B_{i}\right|_{B}
\end{aligned}
$$

where $\mathrm{e}^{\hat{\Xi}_{j} Q_{j}^{i}}$ is the interval matrix exponential which takes into account the uncertainties in the joint angle $\left(Q_{j}^{i}\right)$ and in the joint axis position and direction $\left(\boldsymbol{\Xi}_{j}\right), T_{1}^{i}$ (resp. $T_{2}^{i}$ ) is a constant homogeneous transformation between the reference frames attached to points $P$ and $A_{i}$ (resp. $A_{i}$ and $B_{i}$ ), $\left.C_{i}\right|_{B}$ are the homogeneous coordinates of the point $C_{i}$ expressed in the reference frame attached to point $B_{i}$.

\section{E. Results}

With (11), the process to find $\Theta_{1}$ and $\Theta_{2}$ by the intervals $Q_{i}$ (intervals associated to variables $q_{i}$ ) is directly addressed and the SIVIA algorithm is employed for that. In our case, we identify: $x=\left\{\Theta_{1}, \Theta_{2}\right\}, Y=\left\{Q_{i}\right\}$ and $f^{-1}$ is the inverse kinematic interval model. The INTLAB toolbox of Matlab [19] is used to implement the model and the SIVIA algorithm and to perform the interval calculations.

Fig. $8 \mathrm{a}, 8 \mathrm{~b}$ and $8 \mathrm{c}$ show the solution of each of the three components of (11) (see the black colored area of the figures). Fig. $8 \mathrm{~d}$ is the region that belongs to the intersection of the three solutions of each component of (11). This solution set (black colored area in 8d) contains the kinematically admissible values of $\Theta_{1}$ and $\Theta_{2}$ around the nominal configuration $\theta_{1}=15^{\circ}, \theta_{2}=-15^{\circ}$. In Fig. 8d, the uncertainties of the angle $\Theta_{1}$ and of the angle $\Theta_{2}$ are intervals of amplitude $0.02^{\circ}$ and $0.01^{\circ}$ respectively. These uncertainties in the angles of the microrobot platform will directly affect the projection of the laser spot onto the vocal fold. For instance, if the working distance is $20 \mathrm{~mm}$, the scanning uncertainties, i.e. error of the laser scanning on the targeted fold, are in the order of $14 \mu \mathrm{m}$.

The results shown in Fig. 9 are obtained by running the same algorithm for different sets of coupled angles i.e., $\left(15^{\circ}, 15^{\circ}\right),\left(-15^{\circ}, 15^{\circ}\right),\left(-15^{\circ},-15^{\circ}\right)$ and $\left(0^{\circ}, 0^{\circ}\right)$. These angles correspond to the other extreme tilts (minimum and maximum angles) of the platform of the microrobot, as well as in the central configuration. Each plot in the figure shows the final solution set.

These results show that the uncertainties are large when 
the platform is oriented at $\left(0^{\circ}, 0^{\circ}\right)$. These maximum uncertainties are quantified as $0.10^{\circ}$. When employing the platform to orient a laser beam onto a vocal fold distanced at $20 \mathrm{~mm}$, the related scanning uncertainties are about $70 \mu \mathrm{m}$, which remain acceptable for the targeted phonosurgery task.

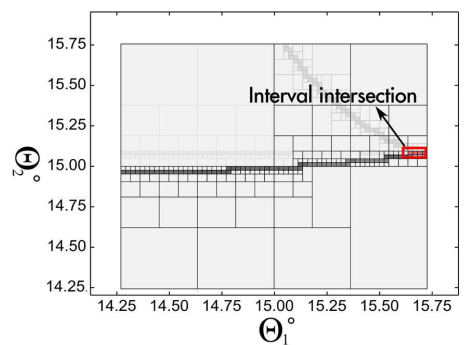

(A)

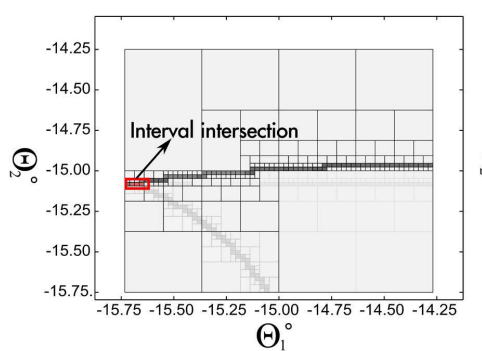

(C)

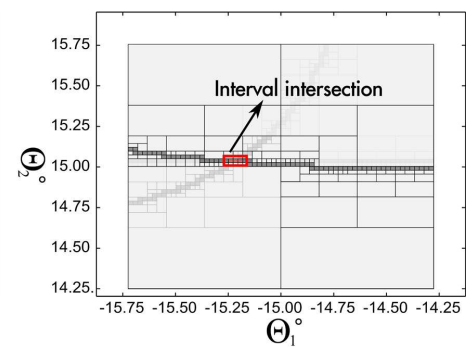

(B)

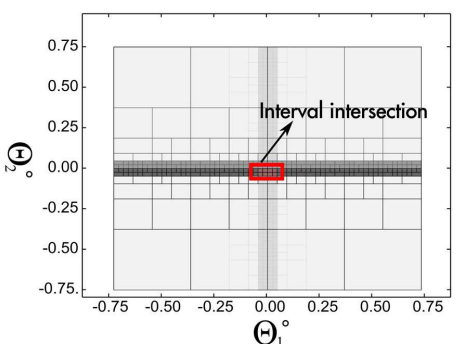

(D)
Fig. 9: Algorithm performed to several angles (A) $\left(\theta_{1}, \theta_{2}\right)=$ $\left(15^{\circ}, 15^{\circ}\right)$ (B) $\left(\theta_{1}, \theta_{2}\right)=\left(-15^{\circ}, 15^{\circ}\right)$ (C) $\left(\theta_{1}, \theta_{2}\right)=$ $\left(-15^{\circ},-15^{\circ}\right)(\mathrm{D})\left(\theta_{1}, \theta_{2}\right)=\left(0^{\circ}, 0^{\circ}\right)$

\section{CONCLUSION AND FUTURE WORKS}

This paper deals with the performances analysis of a novel parallel kinematic microrobot, devoted to laser phonosurgery tasks and capable of performing two orientation angles. Fabricated with the smart composite microstructures (SCM) technique, the microrobot has a complex 3D structure with interesting dexterity performances, but inherits defects in the joints due to the flexibility of the materials. These defects generate uncertainties and imprecision at the output displacements of the microrobot, which may compromise its performances during the execution of the targeted tasks. We therefore proposed to utilize intervals as bounds of these uncertainties and combined interval techniques with the exponential representation of the kinematic of the microrobot to calculate the final imprecision of the latter. We have shown that the maximal error on the angles of the microrobot is of $0.1^{\circ}$, which corresponds to about $70 \mu \mathrm{m}$ of scanning error during the laser phonosurgery task, which is acceptable for the targeted surgery.

The full characterization of the microrobot fabricated with the SCM technology is ongoing which will permit to further verify experimentally the theoretical contribution of this paper. On the other hand, the presented model and precision evaluation in this paper dealt with static performances. Ongoing work also consists in evaluating the precision of the microrobot when the frequency of the laser scanning is high, i.e. when the microrobot is excited at high frequencies (i.e. close to the expected mechanical bandwidth of $200 \mathrm{~Hz}$ ).

\section{ACKNOWLEDGMENT}

Authors would like to thank Dr. Naresh Marturi of AS2M, FEMTO-ST for his support. This work has been supported by the European Union Seventh Framework Programme. Project "Micro-Technologies and Systems for Robot assisted Laser Phonomicrosurgery" called $\mu$ RALP - www.microralp.eu (FP7-ICT-2011-7), and by the French ANR Labex no. ANR11-LABX-01-01 (www.labex-action.fr).

\section{REFERENCES}

[1] D. Jako and S. Strong, "Use of co2-laser in microsurgery of larynx," $H N O$, vol. 22, p. 122, 1974.

[2] "Microtechnologies and systems for robot assisted laser phonomicrosurgery," FP7-ICT-2011-7 european project. [Online]. Available: http://www.microralp.eu/

[3] M. Rakotondrabe, Smart Materials-Based Actuators at the Micro/Nano-Scale: Characterization, Control, and Applications. Springer London, 2013.

[4] S. Lescano, D. Zlatanov, M. Rakotondrabe, and N. Andreff, "Kinematic analysis of a meso-scale parallel robot for laser phonomicrosurgery," in Interdisciplinary Applications of Kinematics. Springer, 2015, pp. 127-135.

[5] C. D. Onal, R. J. Wood, and D. Rus, "An origami-inspired approach to worm robots," Mechatronics, IEEE/ASME Transactions on, vol. 18, no. 2, pp. 430-438, April 2013.

[6] R. Wood, S. Avadhanula, M. Menon, and R. Fearing, "Microrobotics using composite materials: the micromechanical flying insect thorax," in IEEE International Conference on Robotics and Automation, vol. 2, sept. 2003, pp. 1842-1849.

[7] R. J. Wood, S. Avadhanula, R. Sahai, E. Steltz, and R. S. Fearing, "Microrobot design using fiber reinforced composites," Journal of Mechanical Design, vol. 130, no. 5, pp. 052 304+, 2008.

[8] P. Sreetharan, J. Whitney, M. Strauss, and R. Wood, "Monolithic fabrication of millimeter-scale machines," Journal of Micromechanics and Microengineering, vol. 22, no. 5, p. 055027, 2012.

[9] J. Gafford, S. Kesner, R. Wood, and C. Walsh, "Microsurgical devices by pop-up book mems," in Proceedings of the ASME 2013 International Design Engineering Technical Conferences \& Computers and Information in Engineering Conference IDETC/CIE 2013, vol. 6A, Portland, Oregon, USA, 4-7 Aug. 2013.

[10] Z. You, "Folding structures out of flat materials," Science, vol. 345, no. 6197, pp. 623-624, 2014.

[11] M. Tolley, S. Felton, S. Miyashita, D. Aukes, D. Rus, and R. Wood, "Self-folding origami: shape memory composites activated by uniform heating," Smart Materials and Structures, vol. 23, no. 9, p. 094006, 2014.

[12] J.-P. Merlet, "Interval analysis and robotics," in Robotics Research, ser. Springer Tracts in Advanced Robotics, M. Kaneko and Y. Nakamura, Eds. Springer Berlin Heidelberg, 2011, vol. 66, pp. 147-156.

[13] M. Pac and D. Popa, "Interval analysis of kinematic errors in serial manipulators using product of exponentials formula," IEEE Transactions on Automation Science and Engineering, vol. 10, no. 3, pp. 525-535, 2013.

[14] M. R. Pac, M. Rakotondrabe, S. Khadraoui, D. O. Popa, and P. Lutz, "Guaranteed manipulator precision via interval analysis of inverse kinematics," in IDETC/CIE, (International Design Engineering Technical Conference \& Computers and Information in Engineering Conference). ASME, 2013.

[15] J. Selig, Geometric Fundamentals of Robotics, ser. Monographs in Computer Science. Springer, 2010.

[16] J. Merlet, Parallel Robots, ser. Solid Mechanics and Its Applications. Springer, 2006.

[17] A. Goldsztejn, "On the exponentiation of interval matrices," CoRR, vol. abs/0908.3954, 2009.

[18] L. Jaulin and E. Walter, "Set inversion via interval analysis for nonlinear bounded-error estimatimation," Automatica, vol. 29, no. 4, pp. 1053-1064, 1993.

[19] S. Rump, "Intlab interval laboratory," in Developments in Reliable Computing, T. Csendes, Ed. Springer Netherlands, 1999, pp. 77-104. [Online]. Available: http://dx.doi.org/10.1007/978-94-017-1247-7_7 